\title{
High cervical spinal cord stimulation for occipital neuralgia: a case series and literature review
}

\author{
This article was published in the following Dove Press journal: \\ Journal of Pain Research
}

\section{Pavlos Texakalidis \\ Muhibullah S Tora \\ Purva Nagarajan \\ Orion P Keifer Jr \\ Nicholas M Boulis}

Department of Neurosurgery, Emory University School of Medicine, Atlanta, GA, USA
Correspondence: Pavlos Texakalidis Department of Neurosurgery, Emory University School of Medicine, I0I

Woodruff Circle, Atlanta, GA 30322, USA

Tel + I 644706985879

Email pavlostex.med@gmail.com
Background: Occipital neuralgia $(\mathrm{ON})$ is defined as paroxysmal pain in the distribution of the greater, lesser, and/or third occipital nerves. ON can be refractory to conservative management and minimally invasive interventions. Neuromodulatory procedures can potentially treat refractory $\mathrm{ON}$ and include occipital nerve stimulation and the sparsely reported high cervical spinal cord stimulation (SCS).

Objective: To report our experience and conduct a systematic literature review of studies evaluating the effect of high cervical SCS as a treatment modality for refractory ON.

Methods: A retrospective review of patients with refractory ON who underwent high cervical SCS was conducted. In addition, a systematic literature review was performed according to the PRISMA guidelines.

Results: Five patients with refractory ON were treated with high cervical (C1-C3) SCS in our institution. Two out of five (40\%) patients reported a successful trial stimulation $(>50 \%$ pain reduction) and received permanent implantation. During the follow-up, the visual analog scale score decreased from 7.5 to 4 and from 6.5 to 5 in these patients. No complications were reported for any of the patients. The systematic literature review, identified two eligible studies, comprising 18 patients overall who underwent cervicomedullary junction SCS. Nine out of 18 patients $(50 \%)$ had a successful trial and received permanent implantation.

Conclusion: High cervical or cervicomedullary junction SCS is associated with a $40-50 \%$ successful trial rate in refractory ON. No major complications were noted during the followup. Future studies are needed to compare the different neurosurgical options, in order to identify the optimal treatment strategy for refractory $\mathrm{ON}$.

Keywords: occipital neuralgia, spinal cord stimulation, neuromodulation

\section{Introduction}

Occipital neuralgia (ON) is defined by the International Headache Society as paroxysmal shooting, stabbing or sharp pain the distribution of the greater, lesser, and/or third occipital nerves. ${ }^{1}$ The etiology of $\mathrm{ON}$ is considered idiopathic. However, several etiologies including trauma, compression, infection, nerve entrapment, temporal arteritis, schwannoma have been implicated in the etiology of ON. ${ }^{2-4}$ There is a significant overlap in the presentation of $\mathrm{ON}$ and other primary headache disorders including migraines, cluster headaches, hemicrania continua, and tension headaches. Therefore, a detailed history and physical examination are key components in order to distinguish ON from headache syndromes with similar presentations. ${ }^{5}$ Importantly, a characteristic feature of $\mathrm{ON}$ is that local anesthetic block of the affected nerve can temporarily provide pain relief. ${ }^{5}$ However, it is not uncommon for $\mathrm{ON}$ to represent a diagnosis of exclusion. 
First-line conservative treatment of ON includes rest, warm, or cold compress and physical therapy. ${ }^{6}$ Pharmacologic management of chronic ON pain consists of anti-epileptic drugs, gabapentin, pregabalin, tricyclic antidepressants, whereas muscle relaxants and anti-inflammatory medications are usually reserved for the management of acute pain. In cases of resistant $\mathrm{ON}$ to pharmacologic therapy, interventions including occipital nerve blocks with local anesthetics, corticosteroids or Botulinum A toxin injections have been utilized. $5,7,8$ Nevertheless, these are limited by the transience of their analgesic effect. Several neurosurgical interventions have been used for intractable ON including ablative and neuromodulatory procedures. Ablative procedures, which are irreversible, include $\mathrm{C} 1-\mathrm{C} 4$ dorsal cervical rhizotomy, $\mathrm{C} 2$ ganglionectomy, and mechanical or pulsed radiofrequency neurectomy. ${ }^{9-11}$ Neuromodulatory procedures, on the other hand, have the advantage of being reversible and comprise of occipital nerve stimulation and the sparsely reported high cervical spinal cord stimulation (SCS). ${ }^{12,13}$

The aim of this study is to report our experience and conduct a systematic literature review of studies evaluating the effect of SCS as a treatment modality for refractory $\mathrm{ON}$.

\section{Methods}

\section{Study design and patient population}

This was a retrospective study of five patients with refractory ON who underwent high cervical SCS during 20102018 at Emory University Hospital, Atlanta, GA, USA. Medical records were retrieved from the hospital's database after approval of the study protocol by the Institutional Review Board. Written informed consent was obtained. All operations and clinical examination were performed by the senior author (NMB).

The following demographic data were collected: sex, age, body mass index (BMI), diabetes, hypertension, and smoking), duration of symptoms until surgery, analgesic medication used, visual analog scale (VAS) score before and after the procedure, duration of follow-up and complications.

\section{Surgical technique}

At Stage I, a trial stimulation was carried out. Patients underwent percutaneous placement of epidural spinal cord stimulator electrodes in the high cervical region $(\mathrm{C} 1-\mathrm{C} 3)$ for ON for 2 weeks. The following surgical technique was applied for all patients and utilized general anesthesia and endotracheal intubation. Patients are placed in a prone position with their head firmly positioned in the Mayfield. They are then draped and prepared in a sterile fashion. Intraoperative fluoroscopy is then used to identify the interspinous space and the lateral aspect of pedicle of the C3 vertebral body on the side of the patient's symptoms. The skin overlying this region is then infiltrated with lidocaine and $2 \%$ epinephrine. A stab incision is then made with a \#11 blade. A Tuohy needle and stylet are then positioned over the incision just lateral to the pedicle, angled superiorly and medially to target the interspinous space. The needle is advanced under fluoroscopic guidance until piercing the ligamentum flavum. The stylet is then removed, and a flexible wire is advanced beyond the tip of the Tuohy needle to confirm access to the epidural space. The wire is then removed, and a spinal cord stimulator electrode is then advanced under fluoroscopic guidance. The electrode is advanced rostrally until positioned in the high cervical region $(\mathrm{C} 1-\mathrm{C} 3)$ just lateral to midline. Two patients (Patient \#2 and Patient \#3) received leads in a similar fashion with electrodes also covering C3-C8 for concomitant intractable upper extremity pain. Lead positioning is then confirmed using fluoroscopy, and externalized wires were anchored to the skin with Injex anchors and sutures (Figure 1). For permanent implantation in Stage II, patients underwent $\mathrm{C} 1$ and/or C2 laminectomy for paddle lead placement. The wires were connected to a flank or gluteal-implanted pulse generator.

\section{Systematic review}

Search strategy and selection criteria

This systematic review was performed according to the PRISMA (Preferred Reporting Items for Systematic reviews and Meta-Analyses) guidelines. ${ }^{14}$ Systematic searches were conducted in PubMed and Cochrane Central. The keywords used for PubMed were "occipital neuralgia" and "spinal cord stimulation". The search was conducted by two independent investigators (PT, MT) on January 2019. Any disagreements or discrepancies were resolved by consensus. The references of the included studies were also manually reviewed to identify further eligible articles.

A study was included in this systematic review if it fulfilled three predefined criteria: 1) prospective or retrospective observational analyses reporting on patient with ON who underwent high cervical SCS; 2) studies that reported quantitative data on clinical outcomes of interest; 3) studies published up to January 2019. 


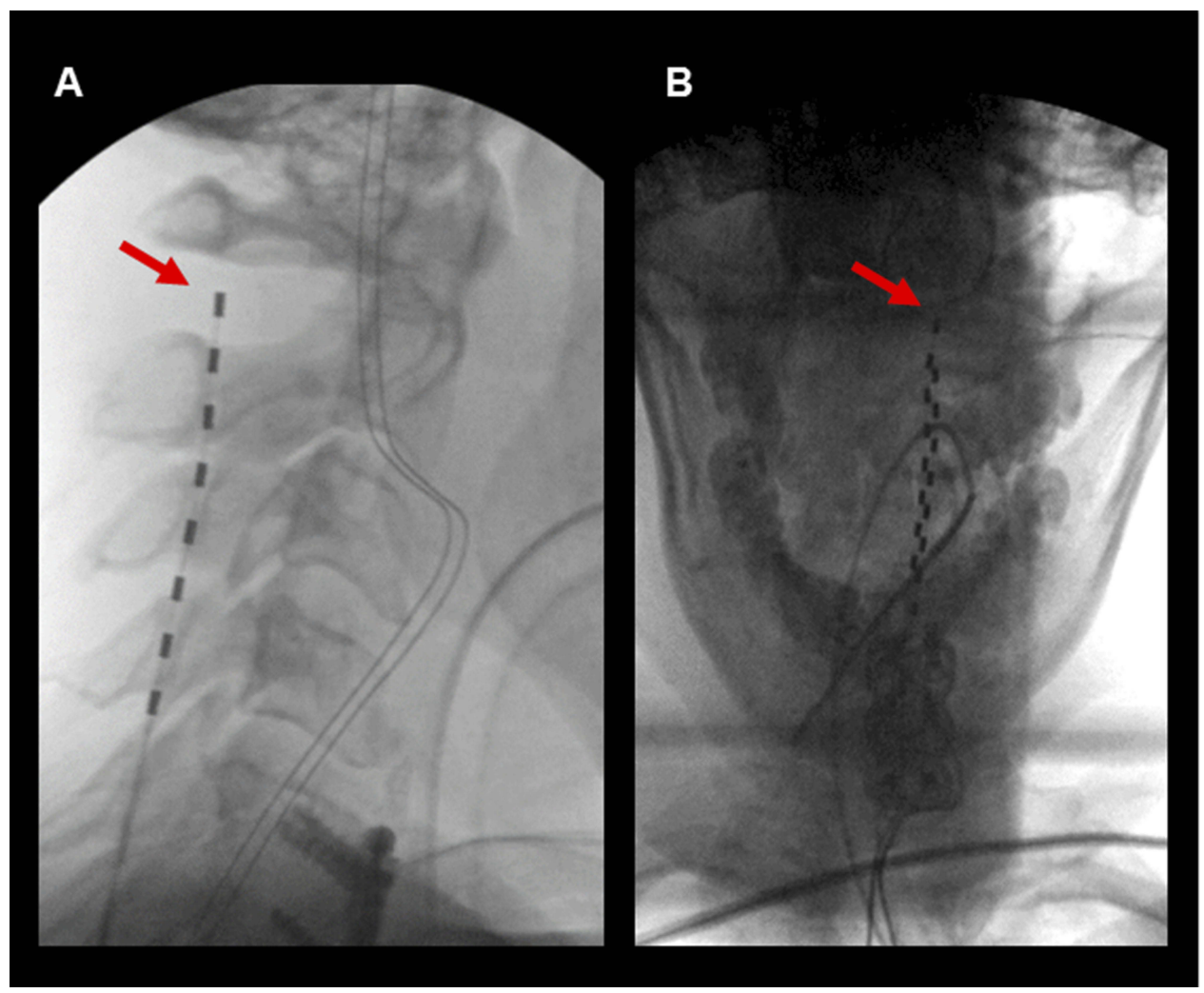

Figure I Intraoperative fluoroscopic lateral image of high cervical lead placement. Lead tip (red arrow).

\section{Data extraction and outcomes}

Two reviewers, blind to each other (PT, MT), independently extracted the relevant data from the eligible studies. Data extracted included the first author, title, date of publication, country of origin, patient number, demographics, history of hypertension, diabetes mellitus, smoking status. The primary endpoint was report of a successful stimulation trial $(>50 \%$ reduction in VAS score before and after the procedure). Secondary outcomes were VAS score reduction during the long-term follow-up for patients who received permanent implantation of the SCS system and complication rates.

\section{Results \\ Present case series}

Five consecutive patients with refractory $\mathrm{ON}$ were treated at our institution. The majority of our patients were female $(n=4 / 5,80 \%)$ with a mean age at presentation 42.7 years and mean BMI was $30.5 \mathrm{~kg} / \mathrm{cm}^{2}$. The average duration of symptoms until surgery was 1.2 years. Patient demographics, analgesic medication used prior to the high cervical SCS and VAS score before the procedure, is summarized in Table 1. SCS was performed in these five consecutive patients due to the following reasons. Two patients (patients \#1 and \#2) received an unsuccessful trial of occipital nerve stimulation prior to the SCS procedure. Patients \#2 and \#3 received SCS from $\mathrm{C} 2-\mathrm{C} 8$ and C1-C8, respectively, in order to address their occipital and concomitant upper extremity pain. Percutaneous trial for patient \#2 could not involve the $\mathrm{C} 1$ level because the patient had previously undergone Chiari decompression with removal of $\mathrm{C} 1$ arch. Lastly, patients \#4 and \#5 received SCS because insurance denied to cover the expenses for a peripheral occipital nerve stimulation trial.

Three patients (patient \#1, 2, and 3) reported that trial stimulation was unsuccessful (VAS reduction $<50 \%$ ). These patients had a history of parieto-occipital meningioma resection, Chiari decompression surgery and cervical spine trauma following a motor vehicle accident. Two out of five patients (patients \#4 and 5) that underwent trial SCS for ON reported $>50 \%$ reduction in their VAS score and therefore, received permanent implantation. Patient \#4 did not report any relevant history/trauma prior to the presentation of the occipital pain, while patient \#5 developed ON 3 months following microvascular decompression for trigeminal neuralgia. During the follow-up (2 and 4.5 months, respectively), the mean reported VAS score 


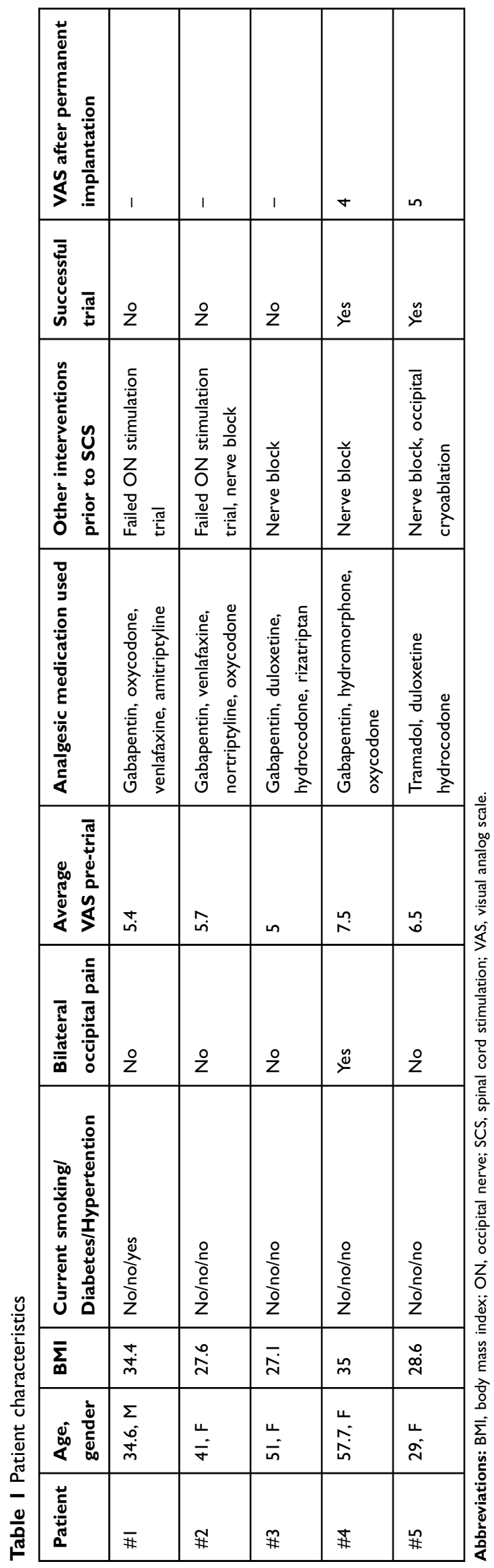

reduced from 7.5 to 4 in patient $\# 4$ and from 6.5 to 5 in patient \#5 with permanent high cervical SCS. No complications were reported following trial or permanent implantation by any of the patients.

\section{Systematic literature review}

A Preferred Reporting Items for Systematic Reviews and Meta-Analysis (PRISMA) search flow diagram is provided in Figure 2. The initial search yielded 15 potentially relevant records after duplicates were removed. After screening of titles and abstracts, seven articles were retrieved for full-text evaluation. Two studies met the predetermined eligibility criteria and were included in the systematic review.

\section{Characteristics of the included studies and patients}

Both studies were observational retrospective cohort analyses including 18 patients overall. ${ }^{13,15}$ Important baseline characteristics of the included studies are presented in Table 2. These studies investigated the effect of cervical or cervicomedullary junction SCS on various head, neck, or extremities pain syndromes. Therefore, baseline characteristics for the various subgroups of patients including those with ON were not specifically reported.

\section{Outcome of interest}

The only available outcome for patients with $\mathrm{ON}$ who underwent SCS was the rate of successful trial. The study by Chivukula et al, reported that $7 / 11$ patients while the study by Tomycz et al, reported that $2 / 7$ patients with $\mathrm{ON}$ had a successful trial (pain reduction $>50 \%$ ). Therefore, a $50 \%(9 / 18)$ rate of successful trial was estimated after combining the included studies.

This rate is in line with the present case series which reported a $40 \%(2 / 5)$ rate of successful trials.

\section{Discussion}

Our institutional results and those of the only two published studies on the use of SCS for ON suggest that it has a $40-50 \%$ successful trial rate and permanent implantation. In addition, the present case series also accompanied by a systematic review, highlights the fact that SCS is an underreported treatment modality for refractory ON.

This is the first study that investigates the efficacy of high cervical (C1-C3) SCS specifically in refractory ON cases. Of note, the studies by Chivukula et al, and Tomycz 


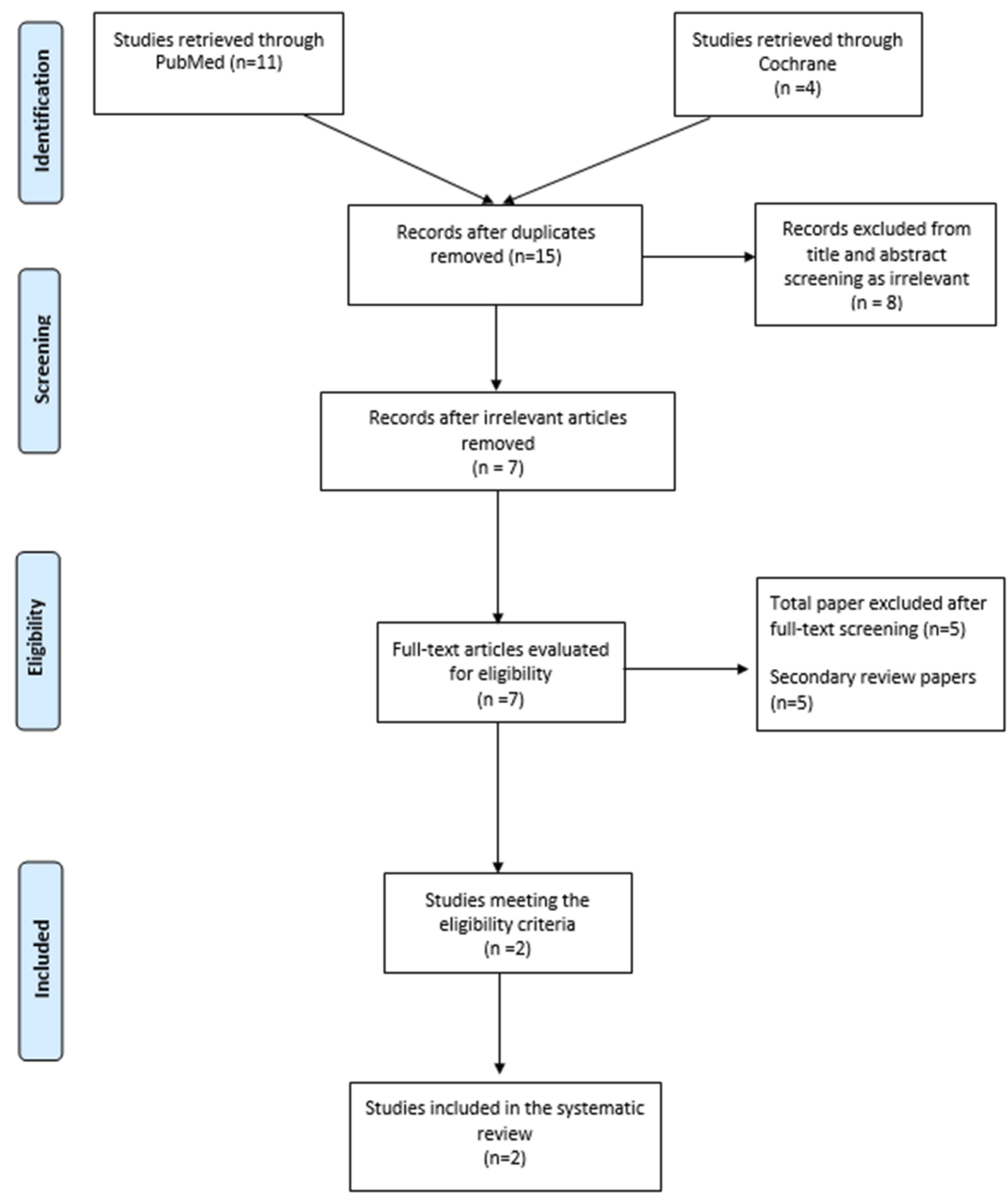

Figure 2 Search PRISMA flow diagram.

Table 2 Included studies in the systematic review

\begin{tabular}{|l|l|l|l|l|l|}
\hline Study & Country & Type of study & Years of enrollment & Number of patients & Successful trials \\
\hline $\begin{array}{l}\text { Chivukula } 2014^{13} \\
\text { Tomycz } 2011^{15}\end{array}$ & USA & Observational & $1991-2014$ & 11 & 7 \\
\\
\hline
\end{tabular}

et al, concluded that cervicomedullary junction SCS had the lowest success rate for occipital pain syndromes as compared to facial pain syndromes. ${ }^{13,15}$ The greater and lesser occipital nerves usually arise from the second spinal nerve (C2 level). A mapping study of sensory responses in humans, showed that epidural stimulation at the $\mathrm{C} 1$ and $\mathrm{C} 2$ levels can generate paresthesias in the lower face, jaw, and occipital region. ${ }^{16}$ However, the same study highlighted that the $\mathrm{C} 1-\mathrm{C} 2$ levels are difficult to cover, as only a small percentage of different electrode arrangements located at the high cervical levels can consistently produce paresthesias. ${ }^{16}$ It is worth highlighting that trial stimulation in the present case series was performed percutaneously because open placement through an occipital 
incision might exacerbate occipital pain; however, lead placement past $\mathrm{C} 1$ or even $\mathrm{C} 2$ can sometimes be challenging and this may impact trial efficacy.

Importantly, no infections, lead migrations, or any other complications occurred in the present case series. Even though surgery at the cervicomedullary junction or high cervical levels can be dangerous by causing significant neurologic morbidity, published studies showed that no major complications occurred other than wound infections, cerebrospinal fluid leak, and lead migrations. ${ }^{13,15}$

Nevertheless, occipital nerve stimulation is a more commonly performed neuromodulatory treatment approach for ON. Several case series, evaluating the efficacy and safety of occipital nerve stimulation, have been published. ${ }^{12,17}$ Based on our experience with occipital nerve stimulation, $83.3 \%$ (20/24) of patients reported a successful trial and underwent permanent implantation. ${ }^{12}$ Notably, the average VAS score reduced from 7.4 before the trial to 2.9 during the long-term follow-up. In addition, occipital nerve stimulation is considered a safer procedure than SCS especially given that the latter involves the high cervical region. It should be noted, however, that implanted stimulation systems are not always MRI compatible which can be a limitation of neuromodulatory treatments. The authors believe that occipital nerve simulation should be the first-line neuromodulatory treatment approach for refractory ON to conservative management and minimally invasive interventions. High cervical SCS should be reserved for resistant ON cases that do not respond to occipital nerve stimulation and other minimally invasive therapies including nerve blocks, radiofrequency ablation, cryoablation, etc. ${ }^{18,19}$ We have also used high cervical SCS in cases of mixed occipital and upper extremity pain, as SCS can potentially treat both. However, notably neither of our patients treated for this reason had successful trials.

A number of limitations should be noted for the present study. First, this was a retrospective non-blinded study. Second, the present case series included a small number of patients. However, this is the first study investigating the effect of high cervical SCS on ON. In contrast, the two studies included in the systematic review, performed cervicomedullary junction stimulation for ON. ${ }^{13,15}$ However, results from these studies and the present case series were similar. Future studies would be needed to directly compare the different neuromodulatory strategies and ablative procedures to identify the optimal approach for refractory $\mathrm{ON}$.

\section{Conclusion}

High cervical or cervicomedullary junction SCS is associated with a $40-50 \%$ successful trial rate in refractory ON. It should be reserved for cases resistant to peripheral nerve stimulation. No major complications were noted during the follow-up. There is a lack of studies evaluating the outcomes of SCS for ON. Future studies are needed to compare the different neurosurgical options, in order to identify the optimal treatment strategy for refractory ON.

\section{Disclosure}

The authors report no conflicts of interest in this work.

\section{References}

1. The international classification of headache disorders, 3rd edition (beta version). Cephalalgia. 2013;33(9):629-808. doi:10.1177/ 0333102413485658

2. Gonzalez-Gay MA, Garcia-Porrua C, Branas F, Alba-Losada J. Giant cell arteritis presenting as occipital neuralgia. Clin Exp Rheumatol. 2001;19(4):479.

3. Ballesteros-Del Rio B, Ares-Luque A, Tejada-Garcia J, MuelaMolinero A. Occipital (Arnold) neuralgia secondary to greater occipital nerve schwannoma. Headache. 2003;43(7):804-807.

4. Kihara T, Shimohama S. Occipital neuralgia evoked by facial herpes zoster infection. Headache. 2006;46(10):1590-1591. doi:10.1111/ j.1526-4610.2006.00616_2.x

5. Dougherty C. Occipital neuralgia. Curr Pain Headache Rep. 2014;18 (5):411. doi:10.1007/s11916-014-0411-x

6. Vanelderen P, Lataster A, Levy R, Mekhail N, van Kleef M, Van Zundert J. Occipital neuralgia. Pain Pract. 2010;10(2):137-144. doi:10.1111/j.1533-2500.2009.00355.x

7. Pfadenhauer K, Weber H. Giant cell arteritis of the occipital arteriesa prospective color coded duplex sonography study in 78 patients. $J$ Neurol. 2003;250(7):844-849. doi:10.1007/s00415-003-1104-2

8. Kapural L, Stillman M, Kapural M, McIntyre P, Guirgius M, Mekhail N. Botulinum toxin occipital nerve block for the treatment of severe occipital neuralgia: a case series. Pain Pract. 2007;7(4):337-340. doi:10.1111/j.1533-2500.2007.00150.x

9. CUSSON DL, KING AB. Cervical rhizotomy in the management of some cases of occipital neuralgia. Guthrie Clin Bull. 1960;29:198208.

10. Wang MY, Levi ADO. Ganglionectomy of C-2 for the treatment of medically refractory occipital neuralgia. Neurosurg Focus. 2002;12 (1):E14. doi:10.3171/foc.2002.12.1.15

11. Jung SJ, Moon SK, Kim TY, Eom KS. A case of occipital neuralgia in the greater and lesser occipital nerves treated with neurectomy by using transcranial doppler sonography: technical aspects. Korean $J$ Pain. 2011;24(1):48-52. doi:10.3344/kjp.2011.24.1.48

12. Keifer OPJ, Diaz A, Campbell M, Bezchlibnyk YB, Boulis NM. Occipital nerve stimulation for the treatment of refractory occipital neuralgia: a case series. World Neurosurg. 2017;105:599-604. doi:10.1016/j.wneu.2017.06.064

13. Chivukula S, Tempel ZJ, Weiner GM, et al. Cervical and cervicomedullary spinal cord stimulation for chronic pain: efficacy and outcomes. Clin Neurol Neurosurg. 2014;127:33-41. doi:10.1016/j. clineuro.2014.09.023

14. Shamseer L, Moher D, Clarke M, et al. Preferred reporting items for systematic review and meta-analysis protocols (PRISMA-P) 2015: elaboration and explanation. BMJ. 2015;349:g7647. doi:10.1136/bmj. g7647 
15. Tomycz ND, Deibert CP, Moossy JJ. Cervicomedullary junction spinal cord stimulation for head and facial pain. Headache. 2011;51 (3):418-425. doi:10.1111/j.1526-4610.2010.01829.x

16. Barolat G, Massaro F, He J, Zeme S, Ketcik B. Mapping of sensory responses to epidural stimulation of the intraspinal neural structures in man. J Neurosurg. 1993;78(2):233-239. doi:10.3171/jns.1993.78.2.0233

17. Liu A, Jiao Y, Ji H, Zhang Z. Unilateral occipital nerve stimulation for bilateral occipital neuralgia: a case report and literature review. $J$ Pain Res. 2017;10:229-232. doi:10.2147/JPR.S125271
18. Manolitsis N, Elahi F. Pulsed radiofrequency for occipital neuralgia. Pain Physician. 2014;17(6):E709-E70917.

19. Kim CH, Hu W, Gao J, Dragan K, Whealton T, Julian C. Cryoablation for the treatment of occipital neuralgia. Pain Physician. 2015;18(3): E363-E368.

\section{Publish your work in this journal}

The Journal of Pain Research is an international, peer reviewed, open access, online journal that welcomes laboratory and clinical findings in the fields of pain research and the prevention and management of pain. Original research, reviews, symposium reports, hypothesis formation and commentaries are all considered for publication. The manuscript

Submit your manuscript here: https://www.dovepress.com/journal-of-pain-research-journal management system is completely online and includes a very quick and fair peer-review system, which is all easy to use. Visit http:// www.dovepress.com/testimonials.php to read real quotes from published authors. 